\title{
CANNABIS, A GROWING PROBLEM: FOR ADOLESCENTS
}

\author{
Lucy Melchert
}

\section{INTRODUCTION}

Cannabis use amongst the adolescent population is a well-established problem that is not adequately addressed. Education for adolescents about substance use generally has a greater focus on alcohol and cigarettes, and little information about the detrimental long-term effects that frequent use of cannabis can have (Clarke et al., 20l4). This is a major issue as Ministry of Health reports show that this age group has the highest rates of past year use of cannabis, as well as being the most strongly represented demographic in studies about the negative mental health effects (Ministry of Health, 2015). Of the negative effects of regular cannabis use, mental health concerns are the most serious and most common. Although research cannot yet definitively determine whether cannabis is a causal factor of psychosis and mental illness, research indicates that cannabis has a significant relationship with psychosis and mood disorders (Hunt, Lenton, \& Witton, 2006). As it is a prominent issue within the adolescent population, there should be an increased focus on developing effective methods of educating adolescents to discourage unsafe habits of cannabis use. As children in New Zealand are legally required to attend school until the age of 16 years (Ministry of Education, 2019), school-based educational programmes appear to be the most effective way to ensure that education is delivered to the majority of New Zealand's adolescents. To do this most effectively, collaboration is required from healthcare professionals and educators. This allows for the utilisation of the health professional's expertise on the health effects of cannabis, and the educator's knowledge of how to most effectively deliver information to the adolescent audience (Porath-Waller, Beasley, \& Beirness, 2010). This review will further discuss the issue that negative mental health effects that cannabis has, specifically for adolecents; as well as exploring recommendations to maximise the effectiveness of educational programmes designed to decrease adolescent cannabis use.

\section{BACKGROUND}

To effectively discourage cannabis use amongst adolescents, there must first be an understanding as to why it is so popular within this demographic. Within the United Nations, cannabis is considered to be the most commonly misused drug (Fergusson \& Boden, 20II), and its popularity continues to remain high amongst youth. Reasons for its popularity could be due to the easy accessibility of the drug and the perception that it is less harmful than cigarettes (Gledhill-Hoyt, Lee, Strote, \& Wechsler, 2000). As well as this, media has a strong influence on the public's attitude towards cannabis as it is one of the public's main sources of information (Gelders, et al., 2009). Media has depicted cannabis more positively within the last few decades and public perception has followed this lead (Stryker, 2003). In addition, mainstream music and music videos, popular amongst adolescents, reference cannabis uses as frequently as 20 percent of the time. This has a direct influence on the general adolescent attitude toward the drug (Primack, Kraemer, Fine, \& Dalton, 2009).

The Canadian Centre on Substance Abuse (2013) carried out a study to understand the general adolescent perception of cannabis. The study revealed that some participants had an accurate impression of the effects of the drug, but many did not. Some participants did not even consider cannabis to be a drug. Common misconceptions 
included the belief that cannabis has positive effects on mental health and helps with sleep and creativity. There were also mixed opinions regarding whether cannabis use improved or impaired driving performance. Many did not think that cannabis is or should be categorised as a drug as it is "safe" and made naturally. Participants of the study expressed that they were confused as to why it is not legal as they perceive it to be safer that alcohol and tobacco (Porath-Waller, Brown, Frigon, \& Clark, 2013). This study demonstrates that adolescents do not have adequate knowledge to make safe and informed decisions and require education regarding the harms of cannabis use and its long-term effects (Porath-Waller, Notarandrea, \& Vaccarino, 2015).

\section{The effects of cannabis}

The continued popularity of cannabis poses a challenge (Clarke, et al., 2014). Within the last two decades there has been increasing evidence to suggest that regular cannabis use can have negative effects on mental health, particularly for adolescents. This may be due to adolescents still being in the process of brain development (Fergusson \& Boden, 20II). The frequent and heavy use of cannabis can alter structural aspects of the developing brain (Porath-Waller, Notarandrea, \& Vaccarino, 2015). The Tetrahydrocannabinol (THC) component of cannabis has been proven to produce acute psychosis causing users to become confused, anxious, agitated and hypomanic. They may also experience hallucinations and delusions. The severity of these symptoms will vary depending on the potency of THC. In a healthy individual without any precursors of mental illness, these symptoms are likely to be experienced short-term. Long-term effects of cannabis are likely to only effect the 10 to 15 percent of adolescent users who use regularly. Within the 10 to 15 percent of regular users, those who have a family history and/or personal history of mental illness are most likely to have long-term consequences (Fergusson \& Boden, 20II).

\section{Cannabis and psychosis}

Many studies have confirmed the existence of the relationship between heavy cannabis use in adolescence and the development of psychosis (Rey \& Tennant, 2002). Commonly cited evidence which suggests a causal relationship, is a study that was conducted in Sweden with over 50,000 participants. Participants were between 18 to 20 years old and were followed up over 15 years. The results of this experiment showed a relationship between cannabis use in adolescence and an increased risk of developing schizophrenia. In addition, the experiment indicated that the regularity and duration of use of cannabis relates to the severity of psychosis (Andreasson, Allebeck, Engstrom, \& Rydberg, 1987). This study was followed up by further studies in Australia, Germany, Greece, and New Zealand (Henquet, Murray, Linszen, \& Van Os, 2005). The results of these studies confirmed the results of the original study. They showed that compared to non-users, individuals who had used cannabis between 10 to 50 times in their adolescence were three times more likely to develop schizophrenia. For those who had used cannabis greater than 50 times, there were six times as many cases. One of the studies showed that those who had regularly used cannabis in their adolescents are two to three times more likely to develop schizophrenia (Rey \& Tennant, 2002). Another study conducted in the Netherlands presented similar results and suggested that if participants had not had exposure to cannabis, the requirement for the treatment of psychosis could have been reduced by 50 percent (Van Os, et al., 2002). Similarly, other studies have suggested that, if cannabis use was removed from the general population of adolescents, eight percent of schizophrenia diagnoses could be prevented (Beer, 2007). The relationship between cannabis and psychosis can be explained by the permanent changes to the brain caused by repeated exposure to the THC component of cannabis. THC works on the cannabinoid receptors in the brain which directly affect the amount of dopamine released. With the excess production of dopamine, schizophrenic symptoms can occur (Beer, 2007).

\section{Cannabis and mood disorders}

In addition to psychosis disorders, evidence suggests that there is a relationship between the frequent use of cannabis and the development of mental health issues such as depression, anxiety and suicidal thoughts (Rey \& Tennant, 2002). Adolescents are the most strongly represented demographic in these findings (Hall, 2006). 
Evidence of this relationship can be seen in studies such as a national population study conducted in Australia in 200I. The results of this study suggested that in non-users of cannabis, six percent had a mood disorder. In contrast, of those with a cannabis dependence, 14 percent were reported to have a mood disorder (Degenhardt, Hall, \& Lynskey, 200I). Another study conducted in Australia followed adolescents over a seven-year period. The results of this study showed that the weekly, or more frequent use of cannabis by secondary school adolescents, doubled their chance of developing anxiety or depression by the age of 20 years. The study also suggested that there was a dose-effect relation between cannabis use and severity of depression and anxiety amongst participants (Patton, Coffey, Carlin, Degenhardt, \& Lynskey, 2002). A Christchurch study also indicated that there is an association between heavy cannabis use in adolescence and the later development of depression and suicide attempts. This study suggests that cannabis use in adolescence directly contributes to mental health outcomes (Fergusson, Horwood, \& Swain-Campbell, 2002).

\section{IMPLICATIONS}

Although not all researchers agree that cannabis is a causal factor of psychosis and mental illness, the studies appear to indicate a strong relationship (Hunt, Lenton, \& Witton, 2006). Heavy cannabis use has been shown to have a triggering effect in predisposed individuals, causing the development or relapse of psychosis, or exacerbation of symptoms. Higher rates of depression and suicidal ideation are also prevalent amongst regular cannabis users (Rey \& Tennant, 2002). As many as 10 to 15 percent of cannabis users report their level of use as heavy and dependent. Cannabis use has a significant impact on the severity of mental health within New Zealand (Fergusson \& Boden, 201I). Data collected by the Canadian Centre on Substance Abuse showed that between the years of 2006 and 2011, adolescents aged 15 to 24 spent the most time in in hospital due to cannabis-related mental and behavioural disorders when compared to other age groups. The data also showed that over these years there was a 40 percent increase in the time spent in hospital for reasons related to cannabis use by this age group (Young \& Jesseman, 2014).

If cannabis use decreased amongst adolescents, severity and treatment required for psychosis and depression may be reduced (Van Os, et al., 2002), as well as the number of hospital stays (Young \& Jesseman, 20I4). This would reduce the demand on mental healthcare resources and impact the workload in some areas of mental health nursing (Young \& Jesseman, 2014). This is particularly beneficial as only 7.6 percent of New Zealand nurses work in the mental health and addictions specialty resulting in it being categorised as "hard to staff" (Ministry of Health, 2016).

\section{RECOMMENDATIONS}

\section{Effective school based educational programmes}

School-based programmes have been shown to have varying success in decreasing adolecent cannabis use depending on the delivery style, the model of the programme and the number of sessions. Research shows that programmes that run for 15 or more sessions (Porath-Waller, Beasley, \& Beirness, 2010) and are directed at adolescents aged 12 to 16 years old are most effective (Norberg, Kezelman, \& Lim-Howe, 2013). This is because cannabis use is usually initiated between the ages of 12 and 16 years old, therefore, implementing effective education programmes for this this age-group may help decrease the initiating of cannabis use by young adolescents (Hotton \& Haans, 2004; Porath-Waller, Beasley, \& Beirness, 2010).

The method of delivery of the education has been shown to have the most significant impact on the success of the programme (Lize, et al., 2017). Interactive and collaborative delivery methods which allow opportunities for participants to engage with others, are significantly more effective than didactic teaching methods (Tobler, et al., 2000). The individual delivering the programme also has an impact. Studies have shown that programmes run by 
teachers often have poorer outcomes than those delivered by healthcare professionals, or people with personal experience. Generally, the training that healthcare professionals have received provide them with better skills to execute the programme effectively, and people with personal experience with drug abuse are perceived, by adolescents, to be more credible sources of information (Porath-Waller, Beasley, \& Beirness, 20I0; Tobler, et al., 2000). Collaboration between healthcare professionals and educators when developing and delivering the educational programme will maximise its effectiveness (Porath-Waller, Beasley, \& Beirness, 20I0).

The model of the programme can also impact its success. Evidence suggests that programmes based on the social influence model are more successful compared to informational or affective models (Tobler, et al., 2000). Other research suggests that a programme based on a combination of the models is most effective as it will address a broader range of risk factors of adolescent cannabis use. The informational model focuses on the harmful consequences of cannabis use and relies on the facts to motivate participants to make anti-drug decisions. For adolescents, the distant prospect of lung disease or a psychotic illness, does not appear to provide enough motivation to significantly impact participant attitudes towards cannabis (Porath-Waller, Beasley, \& Beirness, 20I0). The social influence approach, however, has shown more success (Das, Salam, Arshad, Finkelstein, \& Bhutta, 2016). This model has an increased focus on the effects that cannabis use can have on relationships with peers and family as well as how it will affect the day-to-day life of participants. It uses role models, such as older peers, and emphasises that most people do not use drugs. It also aims to inform adolescents of the consequenses of drug use as well as the benefits of non-use. This model works to increase confidence and develop decision-making skills so that adolescents are able refuse and resist pro-drug influences. Programmes based on this model are typically more interactive and collaberative, and avoid didactic teaching styles (Porath-Waller, Beasley, \& Beirness, 20I0). As there is more evidence suporting the success of programmes based on the social influence model (Tobler, et al., 2000), it is recommended that future programmes should be based on the this model. Programmes, however, may be improved if they incorporate some aspects of the informational model to ensure that adequate knowledge and facts of long-term effects of cannabis use are delivered to adolecents as well as the advantages of the social influence model.

A review of evidence also indicates that it is beneficial for programmes to undergo regular evaluation. This enables a process of ongoing refinement (Porath-Waller, Beasley, \& Beirness, 20I0).

\section{Utilising media}

Research has shown that although school-based programmes are effective, interventions are most effective when several techniques are used collaboratively to deliver a singular, strong message (Ministry of Public Health of Belgium, 2002). A popular method of substance use education which targets a broad audience is the media campaign (Das, Salam, Arshad, Finkelstein, \& Bhutta, 2016). Although there is limited research surrounding the effectiveness of media campaigns on the reduction of adolescent cannabis use (Hunt, Lenton, \& Witton, 2006), media campaigns have been shown to be an effective method of providing tobacco education to adolescents resulting in reduced smoking initiation (Das, Salam, Arshad, Finkelstein, \& Bhutta, 2016). Hunt, Lenton and Witton (2006) state that public health approaches regarding reducing cannabis use amongst adolescents has many parallels to methods used to address tobacco and alcohol use, therefore, media campaigning may be an effective method of providing education to adolescents regarding cannabis use. To use media campaigning most effectively, the campaign should correspond with the school-based educational programme. The collaboration of these two intervention strategies would increase the effectiveness of both interventions as the media campaign will reinforce the knowledge that adolescents learn through their programme (Ministry of Public Health of Belgium, 2002). Research has shown that media campaigning is most effective when research is put into its design so that it appeals to the specified audience. The campaign should also deliver a strong message over a moderate duration (Das, Salam, Arshad, Finkelstein, \& Bhutta, 2016). A media campaign for this purpose should convey consistent messages with the school-based programme. Themes of the messages should coincide with the social intervention model and provide information about the effects of cannabis use on school performance and social relationships as well as 
some information about long-term mental health impacts of heavy cannabis use. Considering the target audience of this media campaign, it would be appropriate to use formats such as posters, flyers and digital media to educate this audience (Thornley \& Marsh, 20I0).

\section{CONCLUSION}

Evidence appears to indicate that regular and heavy cannabis use has a strong relationship with psychosis, depression and suicidal ideations (Rey \& Tennant, 2002). This relationship is most strongly evident in the adolescent population, making the popularity of cannabis use amongst New Zealand adolescents an issue that should be addressed (Ministry of Health, 2016). As indicated earlier in this review, I believe that the most effective method of reducing adolescent cannabis use is through a school-based education programme based on the social intervention model. The programme should be developed and executed collaboratively with healthcare professionals and educators to be most effective (Porath-Waller, Beasley, \& Beirness, 20 I0). Alongside the school-based programme, a concurrent media campaign will also be beneficial to expand and solidify adolescent knowledge on the risks and effects of cannabis use (Ministry of Public Health of Belgium, 2002). The collaborative implementation of these interventions for young adolescents may reduce the rate of cannabis use initiation and improve general adolescent mental health.

Lucy Melchert is a third-year nursing student at Otago Polytechnic. Her interest in the topic of adolescent mental health was motivated though her experiences throughout her study at her mental health placements. She specifically was interested in researching the topic of adolescent substance abuse as it is a prevalent issue in New Zealand that will impact her practice.

Correspondence to: Lucy Melchert, School of Nursing, Otago Polytechnic | Te Kura Matatini ki Otago, Forth Street, Private Bag 1910, Dunedin 9054, New Zealand. Email: lucy.melchert@gmail.com

\section{REFERENCES}

Andreasson, S., Allebeck, P., Engstrom, A., \& Rydberg, U. (1987). Cannabis and schizophrenia. A longitudinal study of Swedish conscripts. Lancet, 1483-1486.

Beer, D. (2007). Cannabis and mental health. Journal of Psychiatric Intensive Care, 3(1), $45-47$.

Clarke, M. C., Coughlan, H., Harley, M., Connor, D., Power, E., Lynch, F., ... Cannon, M. (2014). The impact of adolescent cannabis use, mood disorder and lack of education on attempted suicide in young adulthood. World Psychiatry, 13(3), 322-323.

Das, J. K., Salam, R. A., Arshad, A., Finkelstein, Y., \& Bhutta, Z. A. (2016). Interventions for adolescent substance abuse: An overview of systematic reviews. Journal of Adolescent Health, 59(4), 6I-75.

Degenhardt, L., Hall, W., \& Lynskey, M. (200I). The relationship between cannabis use, depression and anxiety among Australian adults: Findings from the national survey of mental health and well-being. Social Psychiatry and Psychiatric Epidemiology, 36, 219-227.

Fergusson, D. M., Horwood, L. J., \& Swain-Campbell, N. R. (2002). Cannabis use and psychosocial adjustment in adolescence and young adulthood. Addiction, 97, $1123-1135$. 
Fergusson, D., \& Boden, J. (20II). Cannabis use in adolescence. Improving the transition: Reducing social and psychological morbidity during adolescence: A report from the Prime Minister's Chief Science Advisor, 257-27I.

Gelders, D., Patesson, R., Vandonick, S., Steinberg, P., Malderen, S. V., Nicaise, P., ... Laenenc, F. V. (2009). The influence of warning messages on the public's perception of substance use: A theoretical framework. Government Information Quarterly, 26, 349-357.

Gledhill-Hoyt, J., Lee, H., Strote, J., \& Wechsler, H. (2000). Increased use of marijuana and other illicit drugs at US colleges in the 1990s: results of three national surveys. Addiction, 95(II), 1655-1667.

Hall, W. D. (2006). Cannabis use and the mental health of young people. Australian \& New Zealand Journal of Psychiatry, $40(2)$, 105-II3.

Henquet, C., Murray, R., Linszen, D., \& Van Os, J. (2005). The environment and schizophrenia: the role of cannabis use. Schizophr Bulletin, 3I(3), 608-612.

Hotton, T., \& Haans, D. (2004). Alcohol and drug use in early adolescence. Health Reports, 15(3), 9- 19.

Hunt, N., Lenton, S., \& Witton, J. (2006). Cannabis and mental health: Responses to the emerging evidence. Oxford: The Beckley Foundation.

Lize, S. E., lachini, A. L., Tang, W., Tucker, J., Seay, K. D., Clone, S., ... Browne, T. (2017). A Meta-analysis of the Effectiveness of Interactive Middle School Cannabis Prevention Programs. Prevention science: the official journal of the Society for Prevention Research, 18(1), 50-60.

Ministry of Education. (2019, November). Education in New Zealand. Wellington: Ministry of Education.

Ministry of Health. (2015). Cannabis use 2012/2013: New Zealand Health Survey. Wellington: Ministry of Health.

Ministry of Health. (2016). Health of the health workforce 2015. Wellington: Ministry of Health.

Ministry of Public Health of Belgium. (2002). Cannabis 2002 report. Brussels: Ministry of Public Health of Belgium.

Norberg, M. M., Kezelman, S., \& Lim-Howe, N. (2013). Primary prevention of cannabis use: a systematic review of randomized control trials. PLOS ONE, 8(I), I-I5.

Patton, G. C., Coffey, C., Carlin, J. B., Degenhardt, L., \& Lynskey, M. (2002). Cannabis use and mental health in young people: cohort study. The British Medical Journal, 325, I195-1198.

Porath-Waller, A. J., Beasley, E., \& Beirness, D. J. (2010). A meta-analytic review of school-based prevention for cannabis use. Health Education \& Behavior, 37(5), 709-723.

Porath-Waller, A., Brown, J., Frigon, A., \& Clark, H. (2013). What Canadian youth think about cannabis. Ottawa: Canadian Centre on Substance Abuse.

Primack, B. A., Kraemer, K. L., Fine, M. J., \& Dalton, M. A. (2009). Media exposure and marijuana and alcohol use among adolescents. Substance Use and Misuse, 44(5), 722-739.

Rey, J. M., \& Tennant, C. C. (2002). Cannabis and mental health. The British Medical Journal, 325, II83-1 84.

Stryker, J. E. (2003). Articles media and marijuana: A longitudinal analysis of news media effects on adolescents' marijuana use and related outcomes. Journal of Health Communication, 8, 305-328.

Thornley, L., \& Marsh, K. (2010). What works in social marketing to young people? Wellington: Health Research Council of New Zealand and the Ministry of Youth Development.

Tobler, N. S., Roona, M. R., Ochshorn, P., Marshall, D., Streke, A., \& Stackpole, K. (2000). School-based adolescent drug prevention programs: 1998 meta-analysis. The Journal of Primary Prevention, 20, 275-336.

Van Os, J., Bak, M., Hanssen, M., Bijl, R., de Graaf, R., \& Verdoux, H. (2002). Cannabis use and psychosis: A longitudinal populationbased study. Am J Epidemiol, 156, 319-327.

Young, M. M., \& Jesseman, R. A. (2014). The impact of substance use disorders on hospital use. Ottawa: Canadian Centre on Substance Abuse. 